NOTICE: This is the author's version of a work that was accepted for publication in Materials Science and Engineering: C. Changes resulting from the publishing process, such as peer review, editing, corrections, structural formatting and other quality control mechanisms may not be reflected in this document. Changes may have been made to this work since it was submitted for publication. A definitive version was subsequently published in Materials Science and Engineering: C, Volume 33, Issue 8, December 2013, Pages 49524957. http://dx.doi.org/10.1016/j.msec.2013.08.023 


\title{
Characterisation of micro-sized and nano-sized tungsten oxide-epoxy composites for radiation shielding of diagnostic $\mathrm{X}$-rays
}

\author{
Noor Azman N.Z ${ }^{\mathrm{a}, \mathrm{b}}$, Siddiqui S.A $\mathrm{A}^{\mathrm{a}}$, Low I.M $\mathrm{M}^{\mathrm{a}^{*}}$ \\ a Department of Imaging and Applied Physics, Curtin University, GPO Box U1987, Perth, WA \\ 6845 Australia \\ bShool of Physics, Universiti Sains Malaysia, 11800 Penang, Malaysia \\ *Corresponding author: j.low@curtin.edu.au; Tel: +618-9266 7544; Fax: +618-9266 2377
}

\begin{abstract}
Characteristics of X-ray transmissions were investigated for epoxy composites filled with 2-10 vol\% WO 3 loadings using synchrotron X-ray Absorption Spectroscopy (XAS) at 10-40 keV. The results obtained were used to determine the equivalent X-ray energies for the operating X-ray tube voltages of mammography and radiology machines. The results confirmed the superior attenuation ability of nano-sized $\mathrm{WO}_{3}$-epoxy composites in the energy range of $10-25 \mathrm{keV}$ when compared to their micro-sized counterparts. However, at higher synchrotron radiation energies (i.e., 30-40 keV), the X-ray transmission characteristics were similar with no apparent size effect for both nano-sized and micro-sized $\mathrm{WO}_{3}$-epoxy composites. The equivalent $\mathrm{X}$-ray energies for the operating X-ray tube voltages of the mammography unit (25-49 kV) were in the range of 15$25 \mathrm{keV}$. Similarly, for a radiology unit operating at 40-60 kV, the equivalent energy range was 25-40 keV, and for operating voltages greater than $60 \mathrm{kV}$ (i.e., 70-100 kV), the equivalent energy was in excess of $40 \mathrm{keV}$. The mechanical properties of epoxy composites increased initially with an increase in the filler loading but a further increase in the $\mathrm{WO}_{3}$ loading resulted in deterioration of flexural strength, modulus and hardness.
\end{abstract}

Keywords: X-ray shielding, micro-sized $\mathrm{WO}_{3}$-epoxy composites, nano-sized $\mathrm{WO}_{3}$-epoxy composites, $\mathrm{X}$-ray transmission, filler loading 


\section{Introduction}

Hitherto, numerous analytical methods have been developed to investigate the effect of the particle size of a material on the X-ray attenuation for various incoming X-ray energies including scattered gamma-rays and X-rays [1-11]. It is widely believed that nano-sized particles are able to disperse more uniformly within the matrix with less agglomerations when compared to microsized particles, thus improving the X-ray attenuation ability of the material [6,12, 13]. For instance, Hołyńska (1969) found that the intensity of scattered radiations increased with increases in the grain size of a material. This size effect has been observed in a sand matrix and for samples containing heavy elements such as iron or barium [4].

Filler-reinforced polymers have gained increasing attention from X-ray technologists in radiation shielding since polymers have great potential in many important applications by virtue of their unique properties, such as low density, the ability to form intricate shapes, optical transparency, low manufacturing cost and toughness. One of the filler-reinforced polymers commonly used for radiation shielding is lead acrylic [14-16]. Moreover, some researchers have also tried to synthesise nano-sized filler-reinforced polymers for radiation shielding by virtue of the size effect in X-ray attenuation [2, 6, 17]. For instance, a recent study by Botelho et al. [13] revealed that the attenuation for X-ray beams generated from low tube voltages (i.e., 26-30kV) in nanostructured copper oxide $(\mathrm{CuO})$ was better than microstructured $\mathrm{CuO}$. However, no significant difference in attenuation was observed for X-rays generated from higher tube voltages (i.e., 60-102 kV). A similar conclusion on this size effect in X-ray attenuation was made by Kunzel et al. [18] for a nanostructured CuO-polymer system.

In a recent work on WO3-filled epoxy composites [19], we investigated the effect of nanosized and micro-sized $\mathrm{WO}_{3}$ filler-epoxy composites on $\mathrm{X}$-ray attenuation in the X-ray tube voltage range of 22-127 kV generated by a mammography unit and a general radiography unit. The equivalent $\mathrm{X}$-ray energies for the various $\mathrm{X}$-ray tube voltages used were in the range of 17.5-60 keV, which conformed to our expectation since the equivalent X-ray energies for a mammography unit were 17.5, 19.6, 20.2 and $22.7 \mathrm{keV}$ which are the characteristic energies of molybdenum and rhodium; while the equivalent X-ray energy for a radiology unit is about onethird of the X-ray tube voltage used. The results showed that nano-sized $\mathrm{WO}$ з was more effective than micro-sized $\mathrm{WO}_{3}$ in $\mathrm{X}$-ray attenuation only in the low X-ray tube voltage range of 22-35 kV 
but this size effect was not apparent at the higher X-ray operating tube voltage range of 40-120 $\mathrm{kV}$ [19]. Hence, the aim of this work was to verify our previous work on X-ray transmission in WO3-filled epoxy composites by using synchrotron radiations as the X-ray source for the characteristic (monochromatic) X-ray energy range of $10-40 \mathrm{keV}$. The results obtained were compared with those of previous work [19] to determine the equivalent energy range of the previous machines used (a mammography unit and a radiology unit).

\section{Experimental Procedure}

\subsection{Sample preparation}

Nano-sized $(<100 \mathrm{~nm})$ tungsten oxide and micro-sized $(\sim 20 \mu \mathrm{m})$ tungsten oxide $\left(\mathrm{WO}_{3}\right)$ were used as the filler for synthesising $\mathrm{WO}_{3}$-epoxy composites. The former was obtained from SigmaAldrich and the latter (FR251) from Fibreglass and Resin Sales. The particle sizes were provided by Sigma-Aldrich.

Details about the preparation of the $\mathrm{WO}_{3}$-epoxy composite samples are available in [19]. For the current work, the thickness of the samples was set at $2.0 \mathrm{~mm}$. The list of the prepared samples with the different volume percentages of $\mathrm{WO}_{3}$ is shown in Table 1 .

\subsection{Measurements of $X$-ray transmission}

This work was done using the X-ray Absorption Spectroscopy (XAS) beamline at the Australian Synchrotron located in Melbourne. Experiments were carried out in the energy range of 10-40 keV using an Si (311) monochromator and a beam size on the sample of about $0.25 \times$ $0.25 \mathrm{~mm}^{2}$ and a photon flux of about $10^{11} \mathrm{ph} / \mathrm{s}$. Data on X-ray transmission $\left(I / I_{o}\right)$ were collected in an ionisation chamber after the sample, and compared to the incident beam flux. For each sample, 20 readings were recorded at each energy level. To normalise the data collected, readings were also recorded for an empty sample holder. The average $I / I_{o}$ was calculated and plotted as a function of filler loadings $\left(\mathrm{WO}_{3}\right.$ vol\%) for each synchrotron radiation energy. The $\mathrm{X}$ ray transmission $\left(I / I_{O}\right)$ was related to the linear attenuation coefficient $(\mu)$ and the thickness of the samples ( $t$ ) through Equation (1):

$$
\left(I / I_{o}\right)=\exp (-\mu t)
$$

Next, all the samples were examined again with a mammography unit and a radiology unit according to previous experiments [19]. 


\subsection{Powder diffraction}

Powder diffraction (PD) measurements were conducted to identify the crystallite size of the WOз. This was done at the Australian Synchrotron in Melbourne, on the Powder Diffraction beamline. Mythen detectors were used to record the diffraction patterns in the $2 \theta$ range of $0 \circ-60^{\circ}$ at a fixed wavelength of $1.13 \AA$. The crystallite size $(L)$ of the nano-sized WOз was determined using the Scherrer equation as follows (Equation 2):

$$
L=\frac{k \lambda}{(F W H M) \cos \theta}
$$

where $k$ is a constant depending upon the crystal shape and size $(k=0.90), \lambda$ is the wavelength, FWHM is the full-width half maximum of the peak and $2 \theta$ is the diffraction angle of the strongest peak.

\subsection{Flexural tests}

Three-point bending tests were used to determine the flexural strength and modulus of the $\mathrm{WO}_{3}$-epoxy composites containing different loadings of nano-sized and micro-sized $\mathrm{WO}_{3}$. Specimens with dimensions $60 \mathrm{~mm} \times 10 \mathrm{~mm} \times 2 \mathrm{~mm}$ were prepared for the test according to the ASTM D790-03 standard [20] on a universal testing machine (LLOYD Instruments). A minimum of three samples was tested for each composite and the average results were taken. In these measurements, the samples were tested to the applied load with the results calculated by the NEXYGEN Plus software.

\subsection{Indentation hardness test}

The hardness values of polished $\mathrm{WO}_{3}$-epoxy composites were determined using a Rockwell hardness tester with scale $\mathrm{H}$. The hardness measurements were conducted using an indentation load of $588.4 \mathrm{~N}$ and ball diameter of $0.3 \mathrm{~cm}$. Five measurements were conducted for each sample in order to obtain an average value.

\section{Results and Discussion}

\subsection{Characteristics of $X$-ray transmissions}

Since the synchrotron radiations of the XAS beamline contain a large range of energies (i.e., 10-40 keV), the thickness of the samples was set at $2 \mathrm{~mm}$ to ensure that the detector was able to 
obtain a meaningful X-ray transmission reading for the lower energy range without being totally absorbed by the samples. As shown in Fig. 1, there was an obvious difference in X-ray transmissions between the micro-sized $\mathrm{WO}_{3}$-ероху and nano-sized $\mathrm{WO}_{3}$-ероху composites of the same $\mathrm{WO}_{3} \mathrm{vol} \%$ at the energy range of $10-20 \mathrm{keV}$. With a further increase of synchrotron energy to greater than $20 \mathrm{keV}$, there was no difference in X-ray transmissions between these two composites, thus indicating the absence of size effect at play. The results show that for all the $\mathrm{WO}_{3}$ loadings in an epoxy matrix, the ratio of the X-ray transmission of the micro-sized $\mathrm{WO}_{3}-$ epoxy composite $\left(I / I_{o}\right)_{m}$ relative to the nano-sized WO3-epoxy composite $\left(I / I_{o}\right)_{n}$, $\left(I / I_{o}\right)_{m} /\left(I / I_{o}\right)_{n}$ remained at $\sim 1.0$ for the synchrotron energy range of $25-40 \mathrm{keV}$. On the other hand, the ratio $\left(\mathrm{I} / \mathrm{I}_{0}\right)_{\mathrm{m}} /\left(\mathrm{I} / \mathrm{I}_{\mathrm{o}}\right)_{n}$ was $1.15-2.3$ for all $\mathrm{WO}_{3}$ loadings (see Fig. 2). The values determined for $\left(I / I_{o}\right)_{m} /\left(I / I_{o}\right)_{n}$ at these energy ranges indicate that the nano-sized WO-epoxy samples absorbed more low energy X-rays than their micro-sized WO-epoxy counterparts.

Further investigations were conducted to verify our previous results [19] obtained from a mammography unit and a radiography unit. In this investigation, all the same measurements from the previous work were repeated with samples of $2 \mathrm{~mm}$ in thickness. Since a mammography unit generates characteristic X-ray energies of molybdenum (17.5 keV and 19.6 $\mathrm{keV}$ ) or rhodium (20.2 keV and $22.7 \mathrm{keV}$ ), it is much easier to compare with the XAS results (see Fig. 3). In the results presented in Fig. 3, it is clearly shown that the X-ray transmission results for the mammography unit sat between the results of $15-25 \mathrm{keV}$ for the XAS beam energies. In contrast, for the radiography unit, the operated $\mathrm{X}$-ray tube voltages generated a broad spectrum (polychromatic X-ray beam). Thus, the equivalent energies for the $\mathrm{X}$-ray tube voltages of the radiography unit were estimated from the XAS results by superimposing their data together (see Fig. 4). As can be seen in Fig. 4, the X-ray transmissions of samples for X-ray tube voltages of $40-60 \mathrm{kV}$ were sitting between $25-40 \mathrm{keV}$ while the others were sitting above 40 $\mathrm{keV}$. Hence, the $\mathrm{X}$-ray tube voltages of $40-60 \mathrm{kV}$ operated by the radiography unit produced the equivalent $\mathrm{X}$-ray energies in the range of $25-40 \mathrm{keV}$ while the $\mathrm{X}$-ray tube voltages of $\geq 60 \mathrm{kV}$ had an equivalent energy of $\geq 40 \mathrm{keV}$.

Figure 5 shows the comparison of X-ray transmissions for samples with different thicknesses. Samples of $7 \mathrm{~mm}$ thickness were used in our previous study [19], whereas $2 \mathrm{~mm}$ thick samples were used in this study. Fig. 5a and Fig. 5b provide the results for the mammography unit only on samples with 4 vol\% and 6 vol\% loading of $\mathrm{WO}_{3}$ respectively. As shown in these figures, the 
differences in the X-ray transmission between the micro-sized and nano-sized $\mathrm{WO}_{3}$-epoxy became larger for thicker samples (7 mm). In contrast, Fig. 5c shows insignificant differences in $\mathrm{X}$-ray transmission between nano-sized and micro-sized $\mathrm{WO}_{3}$-epoxy composites for radiography tube voltages only for samples with a loading of 4 vol\% $\mathrm{WO}_{3}$. This trend was also observed for all the other loadings (i.e., 2, 6, 8 and 10 vol\% $\mathrm{WO}_{3}$ ). These findings are in good agreement with the work by Künzel and Okuno (2012), which also showed that the grain size effect increased with the increase of the sample thickness at low energy X-ray beams ( $25 \mathrm{kV}$ and $30 \mathrm{kV}$ ) but remained unchanged over the material thickness for higher energy X-ray beams (60 kV) [18].

In general, the photoelectric effect is the most likely interaction to occur within a matter at a lower photon (X-ray) energy range. In this interaction, a photon will transfer its entire energy to an electron in the material on which it impinges. The electron thereby acquires enough energies to free itself from the material to which it is bound and then may undergo single or multiplescattering events with neighbouring atoms. In addition, there is also a slight fluctuation in the probability of emission of Auger electrons and fluorescent photons may form during this interaction. This phenomenon can contribute to the alteration of the mass attenuation coefficient of an element relative to the bulk material when considered over a small range of X-ray energies. The probability of photoelectric interaction is directly proportional to the cube of the atomic number of the absorbing material $\mathrm{Z}^{3}$ and inversely proportional to the cube of the $\mathrm{X}$-ray energy $(1 / \mathrm{E})^{3}$.

Moreover, nano-sized $\mathrm{WO}_{3}$-epoxy composites consist of a higher number of $\mathrm{WO}_{3}$ particles/gram when compared to micro-sized $\mathrm{WO}_{3}$-epoxy composites. Therefore, the distribution of the nano-sized $\mathrm{WO}_{3}$ in the resin should also be different from that presented by micro-sized $\mathrm{WO}_{3}$, thus resulting in a more uniform dispersion in the resin. As a consequence, the chances of an X-ray photon with lower energy to interact and be absorbed by $\mathrm{WO}_{3}$ particles may be higher in nano-sized $\mathrm{WO}_{3}$-epoxy composites than in micro-sized $\mathrm{WO}_{3}$-epoxy composites. Figure 6 shows the back-scattered images of the same loading of $\mathrm{WO}_{3}$ (4 vol\%) within nanosized $\mathrm{WO}_{3}$-epoxy and micro-sized $\mathrm{WO}_{3}$-epoxy composites using the Zeiss Evo 40XVP scanning electron microscope. The $\mathrm{WO}_{3}$ particles were seen to be more closely dispersed in the nano-sized $\mathrm{WO}_{3}$-epoxy composite (Fig. 6a) as compared to its micro-sized counterpart (Fig. 6b). Thus, the probability for the lower energy photons to interact with the $\mathrm{WO}_{3}$ particles and be absorbed is higher for the nano-sized $\mathrm{WO}_{3}$-epoxy composite. 
As the photon energy increases, the photon (X-ray) penetration through the absorbing material without interaction increases and hence, less photoelectric effect relative to the Compton effect occurres. Thus, the X-ray attenuation by the absorbing material decreased since the Compton interaction was weakly dependent on $\mathrm{Z}$ and $\mathrm{E}$ and this interaction only took place between the incident photon and one of the outer shell electrons of an atom in the absorbing material.

In order to discover the $\mathrm{X}$-ray shielding ability of the composites, the results were compared to commercial lead $(\mathrm{Pb})$ sheets (model RAS20 Calibrated Absorber Set) of four different thicknesses (i.e., 0.81, 1.63, 3.18 and $6.35 \mathrm{~mm}$ ) (see Fig. 7) using a radiography unit of tube voltages $40-100 \mathrm{kV}$. The results show that although the lead sheets gave the lowest X-ray transmissions at each tube voltage when compared to all compositions of WO3-epoxy composites, the latter with 10 vol\% of either micro-sized or nano-sized $\mathrm{WO}_{3}$ can be a substitute for $\mathrm{Pb}$ in X-ray shielding by increasing the sample thickness to $\geq 7 \mathrm{~mm}$. Hence, the usage of lead in X-ray shielding can be substituted by $\mathrm{WO}_{3}$ whereby $\mathrm{W}$ is lighter and less toxic compared to $\mathrm{Pb}$.

\subsection{Crystallite size}

The reference for fitting the peaks was taken from the International Centre for Diffraction Data PDF-4+ 2009 database. The wavelength for all of these databases was chosen to be the same as the wavelength of the synchrotron radiation used. The diffraction peaks shown in Fig. 8 belong to monoclinic $\mathrm{WO}_{3}$ (PDF file 00-043-1035). These results indicate that both the microsized $\mathrm{WO}_{3}$ and the nano-sized $\mathrm{WO}_{3}$ were single-phase pure without impurities.

The diffraction patterns were plotted only in the $2 \theta$ range of $9-15^{\circ}$ to clearly show the size difference of the peaks for each micro-sized and nano-sized $\mathrm{WO}_{3}$. As shown in Fig. 8, the broad peaks belonged to nanometer-sized $\mathrm{WO}_{3}$ crystallite whereas the well-defined crystalline peaks belonged to micrometer-sized $\mathrm{WO}_{3}$. The crystallite size determined from the Scherrer equation for nano-sized $\mathrm{WO}_{3}$ was $51.5 \mathrm{~nm}$. These nano-crystallites were significantly smaller than the particle sizes provided by Sigma-Aldrich, thus indicating that at least 2 crystallites were present in each $\mathrm{WO}_{3}$ particle of $100 \mathrm{~nm}$ in size.

\subsection{Mechanical properties}


Figure 9 shows the effect of filler loading on the flexural strength, flexural modulus and Rockwell hardness of the epoxy composites. The flexural strength was found to decrease with increased $\mathrm{WO}_{3}$ filler size while it had little or no effect on flexural modulus. Similar results were reported by Park [21] who observed an increase in flexural strength with decreased particle size in silica-reinforced epoxy composites. However, Moloney et al. [22] reported a negligible effect of particle size on flexural modulus in their epoxy composites filled with silica.

From Fig. 9a, it can be seen that the flexural strength of pure epoxy was $49.9 \mathrm{MPa}$, but increased to a maximum value of $64 \mathrm{MPa}$ for the composite containing 4 vol\% nano-sized $\mathrm{WO}_{3}$. However, a further increase in the filler loading beyond 4 vol\% resulted in a decrease in flexural strength whereby the composite containing 10 vol\% nano-sized $\mathrm{WO}_{3}$ exhibited the lowest flexural strength of 52.6 MPa. Similarly for micro-sized $\mathrm{WO}_{3}$-epoxy composites, the maximum flexural strength was obtained for a filler loading of 2 vol\%. A reduction in flexural strength was again observed when the filler loading was increased beyond 2 vol\% due to non-uniform dispersion of the filler within the matrix. The resultant agglomeration of the fillers acted as stress-concentrators which served to reduce the strength of the composites.

The flexural modulus of the composites increased with an increase in the filler loading for both the nano-sized and micro-sized $\mathrm{WO}_{3}$ which may indicate that the stiffness of these composites obeyed the well-known rule-of-mixtures (Fig. 9b).

Finally, the hardness results presented in Fig. 9c indicate that an increase in the $\mathrm{WO}_{3}$ filler loading resulted in an initial increase in the hardness of the composite, but a further increase in filler loading at $10 \mathrm{vol} \%$ caused a reduction in hardness probably due to the undesirable agglomeration of the fillers. The initial increase in the hardness of the composites observed for both the nano-fillers and the micro-fillers may be attributed to their uniform dispersion within the epoxy matrix, together with their strong interaction with the epoxy chains to form good interfacial bonding.

\section{Conclusion}

The size effect of $\mathrm{WO}_{3}$ particles on the X-ray transmission in nano-sized and micro-sized $\mathrm{WO}_{3}$-epoxy composites has been investigated at various synchrotron radiation energies (i.e., 10$40 \mathrm{keV}$ ). The results presented in this work demonstrated that the size effect on X-ray attenuation was profoundly dependent on the energy of the synchrotron radiations. The particle size effect 
was more pronounced at lower synchrotron radiation energies (10-20 keV) since the X-ray transmission in nano-sized $\mathrm{WO}_{3}$-epoxy composites was less than in their micro-sized counterparts. However, this size effect became insignificant at higher energies of $20-40 \mathrm{keV}$ because the X-ray transmissions in both nano-sized and micro-sized $\mathrm{WO}_{3}$-epoxy composites were very similar. The X-ray transmission results for the mammography unit sat between the results of 15-25 keV for XAS beam energies. Meanwhile, the X-ray transmissions in samples for $\mathrm{X}$-ray tube voltages of $40-60 \mathrm{kV}$ of the radiography unit sat between $25-40 \mathrm{keV}$. In addition, for composites with the same filler loading, but with increasing sample thickness, the size effect in $\mathrm{X}$-ray transmission was most prominent for X-ray tube voltages of $25-35 \mathrm{kV}$, but was negligible at $35-100 \mathrm{kV}$. As the filler loading of the $\mathrm{WO}_{3}$ increased, the mechanical properties showed an initial optimum improvement, but a further increase in the filler loading caused these properties to deteriorate.

\section{Acknowledgements}

The collection of X-ray absorption spectroscopy (XAS) data was funded by the Australian Synchrotron (AS123/XAS5341). We thank Dr. Bernt Johannessen of the Australian Synchrotron and our colleagues Dr. C. Ng and A/Prof. Z. Sun for assistance with XAS data collection. Also, we would like to thank Carolyn Madeley of Breast Assessment Centre, Royal Perth Hospital, Western Australia for giving us the opportunity to use the mammography unit.

\section{References}

[1] C.R. Patra, R. Bhattacharya, D. Mukhopadhyay, P. Mukherjee, Advanced Drug Delivery Reviews, 62 (2010) 346-361.

[2] F. Van Den Heuvel, J.P. Locquet, S. Nuyts, Physics in Medicine and Biology, 55 (2010) 4509-4520.

[3] T. Wang, Z. Liu, M. Lu, B. Wen, Q. Ouyang, Y. Chen, C. Zhu, P. Gao, C. Li, M. Cao, L. Qi, Journal of Applied Physics, 113 (2013) 024314-024318.

[4] B. Hołyńska, Spectrochimica Acta Part B: Atomic Spectroscopy, 24 (1969) 85-93.

[5] A. Granmayeh Rad, H. Abbasi, M.H. Afzali, Physics Procedia, 22 (2011) 203-208.

[6] F. El Haber, G. Froyer, Journal of the University of Chemical Technology and Metallurgy, 43 (2008) 283-290. 
[7] P. Jackson, S. Periasamy, V. Bansal, M. Geso, Australasian Physical \& Engineering Science in Medicine, 34 (2011) 243-249.

[8] X. Huang, M.A. El-Sayed, Journal of Advanced Research, 1 (2010) 13-28.

[9] P.D. Sahare, R. Ranju, S. Numan, S.P. Lochab, Journal of Physics D: Applied Physics, 40 (2007) 759.

[10] A. Popov, SPIE Newsroom 24 (2009) 1-2.

[11] S.-S. Chen, H.-C. Chen, W.-C. Wang, C.-Y. Lee, I.N. Lin, J. Guo, C.-L. Chang, Journal of Applied Physics, 113 (2013) 113704-113710.

[12] M. Steinhart, Angewandte Chemie International Edition, 43 (2004) 2196-2197.

[13] M.Z. Botelho, R. Künzel, E. Okuno, R.S. Levenhagen, T. Basegio, C.P. Bergmann, Applied Radiation and Isotopes, 69 (2011) 527-530.

[14] Lablogic, in: L.S. Limited (Ed.), Lablogic, Sheffield, United Kingdom, 2009.

[15] S. Daren, POLYMICRO Newsletter, 2004.

[16] Wardray, Wardray Premise Ltd., Surrey, United Kingdom.

[17] M. Faccini, C. Vaquero, D. Amantia, J. Nanomaterials, 2012 (2012) 1-9.

[18] R. Künzel, E. Okuno, Applied Radiation and Isotopes, 70 (2012) 781-784.

[19] N.Z. Noor Azman, S.A. Siddiqui, R. Hart, I.M. Low, Applied Radiation and Isotopes, 71 (2013) 62-67.

[20] Annual Book of ASTM Standards Vol. 08.01, ASTM. , 2005.

[21] J.J. Park, Transactions On Electrical And Electronic Materials, 14 (2013) 39-42.

[22] A.C. Moloney, H.H. Kausch, T. Kaiser, H.R. Beer, Journal of Materials Science, 22 (1987) 381-393. 
Table 1: Compositions of $\mathrm{WO}_{3}$-epoxy composites with different volume fractions of filler $\mathrm{WO}_{3}$ and epoxy resin.

\begin{tabular}{cc}
\hline & Composite by volume fraction (vol\%) \\
\hline Filler $\left(\mathrm{WO}_{3}\right)$ & Epoxy resin \\
\hline 2 & 98 \\
4 & 96 \\
6 & 94 \\
8 & 92 \\
10 & 90 \\
\hline
\end{tabular}




\section{Figure captions:}

1. Comparisons of X-ray transmission comparison in nano-sized and micro-sized $\mathrm{WO}_{3}$ epoxy composites for synchrotron radiation generated by the XAS unit for energy of $10-$ $40 \mathrm{keV}$.

2. The X-ray transmission ratio of micro-sized $\mathrm{WO}_{3}$-epoxy composite $\left(I / I_{o}\right)_{m}$ to the X-ray transmission for nano-sized $\mathrm{WO}_{3}$-epoxy composite $\left(I / I_{o}\right)_{n}$, $\left(I / I_{o}\right)_{m} /\left(I / I_{o}\right)_{n}$ for synchrotron energies (10-40 keV)

3. Comparisons of X-ray transmission in nano-sized and micro-sized $\mathrm{WO}_{3}$-epoxy composites for synchrotron radiation generated by the XAS unit for energies of 10-25 $\mathrm{keV}$ and mammography unit tube voltages of $25-49 \mathrm{kV}$.

4. Comparisons of X-ray transmission in nano-sized and micro-sized $\mathrm{WO}_{3}$-epoxy composites for synchrotron radiation generated by the XAS unit for energies of 25-40 $\mathrm{keV}$ and radiography unit tube voltages of $40-100 \mathrm{kV}$.

5. X-ray transmission comparison for different thickness of the sample for a) 4 vol\% of $\mathrm{WO}_{3}$ filler epoxy composites for mammography unit tube voltages of 30, 35 and $49 \mathrm{kV}$; b) 6 vol\% of $\mathrm{WO}_{3}$ filler epoxy composites for mammography unit tube voltages of 30, 35 and $49 \mathrm{kV}$; and c) 4 vol\% of $\mathrm{WO}_{3}$ filler epoxy composites for radiography unit tube voltages of 40,60, 80 and $100 \mathrm{kV}$.

6. SEM images for epoxy composites filled with (a) 4 vol\% nano-sized $\mathrm{WO}_{3}$, and (b) 4 vol\% of micro-sized $\mathrm{WO}_{3}$.

7. X-ray transmission as a function of radiography unit X-ray tube voltage $(40-100 \mathrm{kV})$ for all micro-sized and nano-sized WO3-epoxy composites and commercial lead sheets.

8. Typical powder diffraction patterns for micro-sized and nano-sized $\mathrm{WO}_{3}$ loading in epoxy composites.

9. Mechanical properties of epoxy composites showing : (a) flexural strength as a function of $\mathrm{WO}_{3}$ filler loading; (b) flexural modulus as a function of $\mathrm{WO}_{3}$ filler loading; and (c) Rockwell hardness as a function of $\mathrm{WO}_{3}$ filler loading. 